\title{
Population-based study of the relationship of treatment and sociodemographics on quality of life for early stage breast cancer
}

\author{
Nancy K. Janz ${ }^{1}$, Mahasin Mujahid ${ }^{2}$, Paula M. Lantz ${ }^{3}$, Angela Fagerlin ${ }^{4,5}$, Barbara Salem ${ }^{4}$, Monica \\ Morrow $^{6}$, Dennis Deapen ${ }^{7}$ \& Steven J. Katz ${ }^{3,4,5}$ \\ ${ }^{1}$ Department of Health Behavior and Health Education, School of Public Health, University of Michigan, \\ 1420 Washington Heights, Ann Arbor, MI, $48109-2029$ (E-mail: nkjanz@umich.edu); ${ }^{2}$ Department of \\ Epidemiology, School of Public Health, University of Michigan, 109 Observatory, SPH I, Ann Arbor, MI, \\ 48109-2029; ${ }^{3}$ Department of Health Management and Policy, University of Michigan, SPH II, Room M3116, \\ 109 Observatory, Ann Arbor, MI 48109-2029; ${ }^{4}$ University of Michigan Health System, Department of \\ Internal Medicine, 300 North Ingalls Building, NIB 7C-3A12, Ann Arbor, MI 48109-0429; ${ }^{5}$ Veterans Affairs, \\ Ann Arbor Healthcare System, Ann Arbor, MI; ${ }^{6}$ Department of Surgical Oncology, Fox Chase Cancer \\ Center, Philadelphia, PA; ${ }^{7}$ Department of Preventive Medicine, KECK School of Medicine, University of \\ Southern California
}

Accepted in revised form 28 December 2004

\begin{abstract}
Objective: To examine the relationship between cancer stage, surgical treatment and chemotherapy on quality of life (QOL) after breast cancer and determine if sociodemographic characteristics modify the observed relationships. Methods: A population-based sample of women with Stages 0-II breast cancer in the United States $(\mathrm{N}=1357)$ completed surveys including the European Organization for Research and Treatment of Cancer Quality of Life Questionnaire (EORTC QLQ-C30), and the Breast Cancer-Specific Quality of Life Questionnaire (QLQ BR-23). Regression models calculated mean QOL scores across primary surgical treatment and chemotherapy. Clinically significant differences in QOL were defined as $\geqslant 10$ point difference (out of 100) between groups. Results: Meaningful differences in QOL by surgical treatment were limited to body image with women receiving mastectomy with reconstruction reporting lower scores than women receiving breast conserving surgery $(p<0.001)$. Chemotherapy lowered QOL scores overall across four QOL dimensions ( $p$ values $<0.001$ ), with a disproportionately greater impact on those with lower levels of education. Younger women reported lower QOL scores for seven of nine QOL dimensions ( $p$ values < 0.001). Conclusions: Women should be reassured that few QOL differences exist based on surgical treatment, however, clinicians should recognize that the impact of treatment on QOL does vary by a woman's age and educational level.
\end{abstract}

Key words: Breast cancer, Quality of life, Psychosocial

\section{Introduction}

Treatment for early stage breast cancer consists of surgery, followed by some combination of radiation, chemotherapy, and/or hormone therapy; with each treatment having the potential to alter quality of life (QOL). The majority of women with early stage breast cancer have three surgical choices that carry the same survival: breast conserving surgery (BCS) with radiation, mastectomy only, and mastectomy with reconstruction $[1,2]$. Treatment choices may be related to perceived differences in QOL among the procedures when survival is equivalent. Interest is increasing on the impact 
of breast cancer stage and these various treatment approaches on QOL, and if it differs across subpopulations.

The differential QOL impact dependent on cancer stage at diagnosis has not been well investigated, especially comparing women with invasive vs. non-invasive disease. Amichetti et al. [3] found that while women with ductal carcinoma in situ (DCIS) treated with BCS expressed good physical and social well-being, about $50 \%$ still reported a medium level of depression and anxiety at 4 years follow-up. More recently, Rakovitch et al. [4] compared risk perception and psychological morbidity in women with DCIS vs. invasive disease and found no differences in perception of recurrence and similar levels of psychological distress in the two groups.

Studies that have focused on surgery, have reported few treatment-related differences in QOL between BCS and mastectomy [5-10] with the exception of less impact on body image with BCS $[6,10-15]$. Some studies have noted fewer sexual problems with BCS $[6,11,12,14,15]$, while others report less fear of recurrence with mastectomy [16, 17, and N.K. Katz, personal correspondence]. The impact of mastectomy on body image may be reduced in women who undergo breast reconstruction. Unfortunately, most studies that have compared the QOL impact between BCS and mastectomy have not had sufficient sample sizes to examine outcomes between women who received mastectomy only from those who received mastectomy with reconstruction. Findings have been mixed in studies that addressed reconstruction. For example, Ganz et al. [9] found no significant differences between mastectomy only and mastectomy with reconstruction. Schover and colleagues [7] found no differences between mastectomy with reconstruction and $\mathrm{BCS}$, while Nissen et al. [18] found that those who received breast reconstruction reported lower scores on physical, emotional, and functional well-being compared to those who received mastectomy only. In a large study of women from two metropolitan areas, Rowland et al. [15] found that women who received mastectomy with or without reconstruction did not differ from those receiving BCS on emotional, social or role function, but did report more physical symptoms. Additionally, women with reconstruction were significantly more likely to report that breast cancer had a negative impact on their sex lives.

Fewer studies have focused on the QOL impact of treatment options beyond the primary surgical procedure. The increasing use of adjuvant chemotherapy in women who obtain small incremental survival benefits $[19,20]$ requires an understanding of the QOL impact of this treatment to fully inform women of the risks and benefits. For example, Schover et al. [7] found that after 4 years, women who underwent chemotherapy had more dysfunction, worse body image, and more psychological distress. Ganz et al. [9] found that both physical and sexual functioning were worse for women who received chemotherapy, independent of the type of surgery performed.

The impact of breast cancer treatment on population subgroups has usually involved a focus on one or a limited number of sociodemographic characteristics. Some studies have examined age differences [21-23], and other demographic characteristics, for example race differences [22, 24, 25]. However, most publications are based on relatively small, homogeneous convenience samples which limit their power to examine a variety of sociodemographic characteristics simultaneously. Furthermore, QOL is assessed differently reducing comparability between studies. Although time from treatment is an important independent variable [12], many studies have not controlled for wide variations in elapsed time since surgery within studied groups. This study seeks to address some of the gaps in the literature by using a large population-based sample that over-sampled for women with DCIS and for African American women in order to focus on answering the following questions.

1. Is there a relationship between cancer stage at diagnosis and various dimensions of QOL independent of breast cancer treatment received?

2. What is the relationship of primary surgical treatments for breast cancer on various dimensions of QOL, and does it differ between women who received mastectomy only and those who received breast reconstruction?

3. What is the relationship of chemotherapy (for women with Stage I and II) and radiation therapy on QOL and does it differ depending on initial surgical treatment? 
4. Are sociodemographic characteristics associated with QOL, and do they modify the observed relationships between treatment for breast cancer and QOL?

\section{Methods}

\section{Study population}

The study population consisted of women diagnosed with DCIS and invasive, nonmetastatic breast cancer in Detroit, Michigan and Los Angeles, California. Eligibility criteria included: (1) age $\leqslant 79$ years, (2) a primary diagnosis of breast cancer treated with a definitive surgical procedure, (3) residing in the catchment area of the Detroit and Los Angeles Surveillance Epidemiology and End Results (SEER) sites and, (4) capable of completing a written or telephone survey in English or Spanish. Only women who completed the written survey were included as the telephone survey lacked the primary QOL measures. Asian women in Los Angeles were also excluded due to participation in competing studies. Women were also excluded who had: a previous history of primary breast cancer; presented with metastatic disease; or, had a diagnosis of lobular carcinoma in-situ.

\section{Data collection procedures}

Women were identified from SEER registries in Detroit and Los Angeles between December 2001 and January 2003. Most cases of breast cancer were identified within 6 weeks of initial diagnosis. All cases of DCIS and an approximate $20 \%$ random sample of invasive cases were selected in both cities. African American women with invasive disease were over sampled to increase their representation to approximately one-third in Detroit and $20 \%$ in Los Angeles.

Once SEER registries identified potential participants, physicians were notified of our intent to contact patients and less than $1 \%$ were excluded because of physician concerns. An introductory letter was sent to potential subjects followed by a phone call to assess eligibility. Questionnaires and a $\$ 10$ gift were mailed to all who agreed to participate over the phone and to women who could not be reached by phone (approximately 7\% of potential respondents). The Dillman method was used to encourage response rates [26], involving a postcard reminder for non-respondents at 2 weeks, a second letter and survey at 6 weeks, and a follow-up phone call at 10 weeks. At the followup phone call non-responders were offered, if they preferred, a short telephone survey.

After removing identifying information, completed surveys were forwarded to the University of Michigan investigators for data entry and analysis. Subsequently, SEER pathology and survey data were merged using a unique patient identification number. The study protocol was approved by the Institutional Review Boards of the University of Michigan, Wayne State University, and the University of Southern California.

\section{Study measures}

Sociodemographic characteristics: Survey items included age at breast cancer diagnosis, race, education level, employment status, income, and marital status.

Prior health status: The standard self-rated health question was modified as follows: "Before you were told you have breast disease, would you say your health was: poor, fair, good, very good or excellent." Self-rated health has been strongly and independently associated with morbidity and mortality [27]. We also asked about the presence of six chronic conditions: chronic bronchitis, heart disease, diabetes, high blood pressure, stroke, and arthritis.

Clinical factors: Breast cancer stage was classified using the American Joint Committee on Cancer (AJCC) TNM Staging System for Breast Cancer and SEER summary data. Only women with Stages 0-II were included.

Breast cancer treatment: Participants were asked the type of surgical treatment received: breast conserving surgery, mastectomy only, and mastectomy with breast reconstruction.

Additional treatments: Survey items inquired about ongoing or completed treatment including chemotherapy, radiation therapy, and/or hormone (endocrine) therapy.

Missing self-reported information was updated using SEER summary information for the following measures: age at diagnosis, race, marital 
status, response time from diagnosis, primary surgical treatment, chemotherapy, and radiation therapy.

Health-related quality of life: The European Organization for the Research and Treatment of Cancer Quality of Life Questionnaire (EORTC QLQ-C30) [28] and the Breast Cancer-Specific Quality of Life Questionnaire (QLQ BR-23) [29] were used to assess QOL. The EORTC QLQ-C30 version 2 contains 30 questions addressing: five functional domains; one global QOL item; three symptom scales; and, six single items assessing financial impact and physical symptoms using "the past week" as a time frame. The format utilized yes/no, 4-point response, and visual analogue scales. The QLQ-BR23 consists of 23 items including two functional scales on body image and sexual functioning. The body image subscale inquires about feeling less attractive, feeling less feminine, difficulty looking at oneself naked, and dissatisfaction with ones body. The sexual functioning scale asks (over the past month) about interest in sex, and the extent to which women were sexually active, with or without intercourse. The QLQ-BR23 also contains three symptom scales and three single items. The psychometric properties of the EORTC QLQ-C30 [30-33] and the QLQ-BR23 [29] have been previously established with excellent reliability and validity and have been used with breast cancer patients [34-35].

Consistent with recommendations by Sprangers [29], a limited set of subscales and items of prime interest were identified a priori. Nine QOL measures were chosen from the two instruments including: physical, social, emotional, and role functional domains and domains relevant for women being treated for breast cancer (fatigue, body image, future perspective, sexual functioning, and sexual enjoyment). Using the EORTC QLQ-C30 scoring algorithm all QOL measures range from 0 to 100 . Higher scores represent a higher level of functioning for functional measures and a lower level for symptom measures. Because fatigue was the only symptom measure examined in this study, for ease of interpretation, we transformed fatigue scores such that higher scores always indicate better QOL.

A major advantage of the EORTC QLQ-C30 is the considerable work that has been published on interpretation of clinically relevant difference scores [36-37]. Osoba et al. [37] found that patients with breast and lung cancer who perceived a "moderate change either better or worse" over time also reported a corresponding mean change of 10-20 points on their EORTC QLQ-C30 scores. Based on that study, we set the criteria of a between-group difference of 10 points or more to represent a "clinically meaningful difference." [37].

\section{Statistical methods}

Each QOL measure was standardized into a score of 0 to 100 by using a linear transformation as instructed by the EORTC QLQ-30 Scoring Manual. The reliability of all scales was assessed by using Cronbach's alpha coefficient.

Bivariate associations were investigated between stage and treatment and each of the QOL measures, using Analysis of Variance (ANOVA). Multivariable models were analyzed using Analysis of Covariance (ANCOVA) to test for adjusted associations among treatment, sociodemographic characteristics, and QOL measures. Models were constructed with each QOL measure as separate outcomes and included all sociodemographic variables, prior health status, clinical, stage, and treatment variables. Sample weights were included to adjust for design effects resulting from our differential sampling across race and stage. Interactions between treatment and sociodemographic characteristics were assessed separately and retained if the $p$ value $<0.05$. All significant interactions were assessed simultaneously in the final model and only interactions that maintained significance $(p<0.05)$ were retained. Of primary interest were results that were both statistically significant and clinically meaningful, based on the Osoba et al. 10 point criteria [37]. Because all hypotheses were specified a priori no adjustments were made for multiple comparisons. However, a $p$ value $\leqslant 0.001$ can be viewed as statistically significant by the most conservative methods of adjustments for multiple comparisons. All analyses were performed using SAS version 8.2.

\section{Results}

We accrued 2640 cases prospectively into the preliminary study sample. Approximately $88 \%$ 
of cases initially identified by the registries were eligible and $74.3 \%$ of those eligible completed the survey $(93 \%$ completed the written survey and $7 \%$ an abbreviated telephone survey). Nonrespondents were older, more likely to be nonwhite, and more likely to have advanced stage. Nonrespondents were also somewhat more likely to receive mastectomy ( $35 \%$ vs. $31 \%$ ), although the difference was not statistically significant $(p=0.06)$. We excluded 141 women with late stage disease (Stage III, IV), and 184 women who had not completed primary treatment (surgery, chemotherapy, and radiation therapy), of the 1682 eligible participants. Those who were excluded for late stage disease or because they had not completed treatment were more likely to be younger, African American, and never married. The final sample for this study included 1357 women with Stage 0-II breast cancer $(96 \%$ completed the survey in English and $4 \%$ in Spanish).

Table 1 describes characteristics of the study participants overall, and by primary surgery. The mean age of the subjects was 60.0 years (range 27.9-79.9), with $68 \%$ Caucasian, $19 \%$ African American, and 13\% "other" (80\% Hispanic). Average co-morbidities reported was 1.1 and over half of the women reported a very good or excellent health status at diagnosis. Using AJCC criteria, $44 \%$ of women had Stage $0,47 \%$ had Stage I, and $27 \%$ had Stage II breast cancer. Approximately $68 \%$ of women received BCS, $18 \%$ received mastectomy only, and $13 \%$ had mastectomy with reconstruction. About $21 \%$ of women in the final sample completed chemotherapy $(34.0 \%$ with Stage I and II breast cancer), $57 \%$ completed radiation therapy, and 52\% started on hormone therapy. Women who received mastectomy with reconstruction were younger, more educated, and reported few comorbidities than women receiving BCS or mastectomy only. Women who had either BCS or mastectomy with reconstruction were more likely to be white, have higher income, and lower cancer stage at diagnosis than those women who had mastectomy only.

The internal reliability of six QOL outcomes (emotional, role, social, and sexual functioning, fatigue, and body image) yielded a Cronbach's alpha $\geqslant 0.80$, with physical functioning somewhat lower $($ alpha $=0.70)$. Sexual enjoyment and future perspective were single item measures.

ANCOVA models were used to determine the mean scores for the nine QOL outcomes by cancer stage at diagnosis, controlling for study site, prior health status, primary surgical treatment received, response time since treatment, and other treatments received. The results revealed no clinically meaningful differences in any of the QOL outcome scores when comparing Stage 0 vs. Stage I-II. All further analyses exploring the relationship of various aspects of treatment to QOL controlled for cancer stage at diagnosis.

Figure 1 presents results of ANCOVA models used to determine the mean scores for the nine QOL outcomes by primary surgical treatment received, controlling for all other factors. A meaningful difference in QOL was observed in body image with those who received BCS reporting higher scores on body image than those receiving mastectomy with reconstruction. No other clinically meaningful differences were observed by primary surgical treatment, although women with reconstruction had lower QOL scores on all measures except sexual functioning and sexual enjoyment. No meaningful differences were detected in QOL dependent on receipt of radiation (not shown).

Figure 2 displays mean scores for the QOL dimensions among women with Stage I or II breast cancer who received chemotherapy vs. no chemotherapy. Using ANCOVA models, four meaningful QOL differences were identified. Women who received chemotherapy compared to no chemotherapy reported lower QOL scores on social well being, fatigue, body image, and future perspective.

Table 2 describes QOL scores after breast cancer according to selected sociodemographics with bolded numbers representing statistically significant and clinically meaningful results. Age at diagnosis was statistically significant and clinically meaningful for six of nine QOL outcomes. Except for sexual functioning and sexual enjoyment, women over 70 reported higher scores than younger women, while women under 70 reported greater sexual functioning and more sexual enjoyment. For role functioning African American women reported lower scores than white women, and for sexual enjoyment, respondents who were divorced, widowed, 
Table 1. Sample characteristics overall and by primary surgical treatment $(\mathrm{N}=1357)$

\begin{tabular}{|c|c|c|c|c|c|c|}
\hline & $\mathrm{N}$ & $\begin{array}{l}\text { Overall } \\
(\%)\end{array}$ & $\begin{array}{l}\text { Breast conserving } \\
\text { surgery }(\mathrm{N}=928) \\
(\%)\end{array}$ & $\begin{array}{l}\text { Mastectomy only } \\
(\mathrm{N}=238) \\
(\%)\end{array}$ & $\begin{array}{l}\text { Mastectomy } \\
\mathrm{w} / \text { breast reconstruction } \\
(\mathrm{N}=182) \\
(\%)\end{array}$ & $p$-value \\
\hline \multicolumn{7}{|l|}{ Sociodemographic } \\
\hline \multicolumn{7}{|l|}{ Study site } \\
\hline Los Angeles & 712 & 52 & 54 & 54 & 44 & 0.05 \\
\hline Detroit & 645 & 48 & 46 & 46 & 56 & \\
\hline \multicolumn{7}{|l|}{ Age } \\
\hline Mean $(\mathrm{SD})^{\mathrm{a}}$ & 1357 & $60.0(11.3)$ & $60.7(11.1)$ & $62.8(10.4)$ & $52.8(10.4)$ & $<0.0001$ \\
\hline$<50$ yrs & 281 & 21 & 19 & 13 & 41 & \\
\hline $50-70 \mathrm{yrs}$ & 777 & 57 & 58 & 59 & 53 & \\
\hline$>70 \mathrm{yrs}$ & 299 & 22 & 23 & 28 & 6 & \\
\hline \multicolumn{7}{|l|}{ Race } \\
\hline Caucasian & 915 & 68 & 70 & 54 & 76 & $<0.0001$ \\
\hline African American & 256 & 19 & 18 & 28 & 13 & \\
\hline Other & 180 & 13 & 12 & 18 & 11 & \\
\hline \multicolumn{7}{|l|}{ Education } \\
\hline $\begin{array}{l}\text { Grade school/some } \\
\text { high school }\end{array}$ & 162 & 12 & 10 & 23 & 7 & $<0.0001$ \\
\hline High school diploma & 299 & 22 & 23 & 26 & 12 & \\
\hline Some college & 508 & 38 & 39 & 33 & 40 & \\
\hline $\begin{array}{l}\text { College Graduate } \\
\text { and beyond }\end{array}$ & 375 & 28 & 28 & 18 & 41 & \\
\hline \multicolumn{7}{|l|}{ Employed } \\
\hline Yes & 633 & 48 & 48 & 33 & 65 & $<0.0001$ \\
\hline No & 696 & 52 & 52 & 67 & 35 & \\
\hline \multicolumn{7}{|l|}{ Income } \\
\hline$<\$ 20,000$ & 267 & 20 & 18 & 32 & 11 & $<0.0001$ \\
\hline$\$ 20,000-\$ 49,999$ & 381 & 28 & 28 & 33 & 23 & \\
\hline$>=\$ 50,000$ & 560 & 41 & 42 & 24 & 59 & \\
\hline Unknown & 149 & 11 & 12 & 11 & 7 & \\
\hline \multicolumn{7}{|l|}{ Marital status } \\
\hline $\begin{array}{l}\text { Currently married/ } \\
\text { domestic partner }\end{array}$ & 807 & 59 & 60 & 51 & 68 & 0.02 \\
\hline $\begin{array}{l}\text { Divorced/separated/ } \\
\text { widowed }\end{array}$ & 445 & 33 & 32 & 40 & 26 & \\
\hline Never married & 105 & 8 & 8 & 9 & 6 & \\
\hline \multicolumn{7}{|l|}{ Prior health status } \\
\hline Number of co-morbidities ${ }^{a}$ & 1354 & $1.1(1.1)$ & $1.0(1.1)$ & $1.4(1.2)$ & $0.8(1.0)$ & $<0.0001$ \\
\hline \multicolumn{7}{|c|}{ Health status at diagnosis } \\
\hline Poor/fair & 166 & 12 & 11 & 22 & 6 & $<0.0001$ \\
\hline Good & 443 & 33 & 34 & 40 & 23 & \\
\hline Very good/excellent & 729 & 55 & 55 & 38 & 71 & \\
\hline \multicolumn{7}{|l|}{ Clinical factors } \\
\hline \multicolumn{7}{|l|}{ Stage } \\
\hline Stage 0 & 555 & 44 & 44 & 35 & 56 & $<0.0001$ \\
\hline Stage I & 462 & 37 & 40 & 35 & 25 & \\
\hline Stage II & 239 & 19 & 16 & 30 & 19 & \\
\hline Time from treatment ${ }^{\mathrm{a}}$ & 1284 & $7.1(2.6)$ & $7.1(2.5)$ & $7.1(2.7)$ & $6.8(2.8)$ & 0.2 \\
\hline \multicolumn{7}{|l|}{ Treatment } \\
\hline \multicolumn{7}{|l|}{ Chemotherapy $^{\mathrm{b}}$} \\
\hline No & 1064 & 79 & 82 & 73 & 78 & 0.008 \\
\hline Yes & 277 & 21 & 18 & 27 & 22 & \\
\hline \multicolumn{7}{|l|}{ Radiation therapy ${ }^{\mathrm{b}}$} \\
\hline No & 580 & 43 & 21 & 87 & 96 & $<0.0001$ \\
\hline Yes & 776 & 57 & 79 & 13 & 4 & \\
\hline
\end{tabular}


Table 1. Continued

\begin{tabular}{|c|c|c|c|c|c|c|}
\hline & $\mathrm{N}$ & $\begin{array}{l}\text { Overall } \\
(\%)\end{array}$ & $\begin{array}{l}\text { Breast conserving } \\
\text { surgery }(\mathrm{N}=928) \\
(\%)\end{array}$ & $\begin{array}{l}\text { Mastectomy only } \\
(\mathrm{N}=238) \\
(\%)\end{array}$ & $\begin{array}{l}\text { Mastectomy } \\
\mathrm{w} / \text { breast reconstruction } \\
(\mathrm{N}=182) \\
(\%)\end{array}$ & $p$-value \\
\hline \multicolumn{7}{|c|}{ Hormone therapy ${ }^{c}$} \\
\hline No & 648 & 48 & 47 & 50 & 55 & 0.09 \\
\hline Yes & 691 & 52 & 53 & 50 & 45 & \\
\hline
\end{tabular}

${ }^{\mathrm{a}}$ Mean $(\mathrm{SD}=$ standard deviation) reported for continuous variables.

${ }^{\mathrm{b}}$ For chemotherapy and radiation therapy yes indicates number of women who have completed treatment.

${ }^{\mathrm{c}}$ For hormone therapy yes indicates number of women who have started or finished treatment.

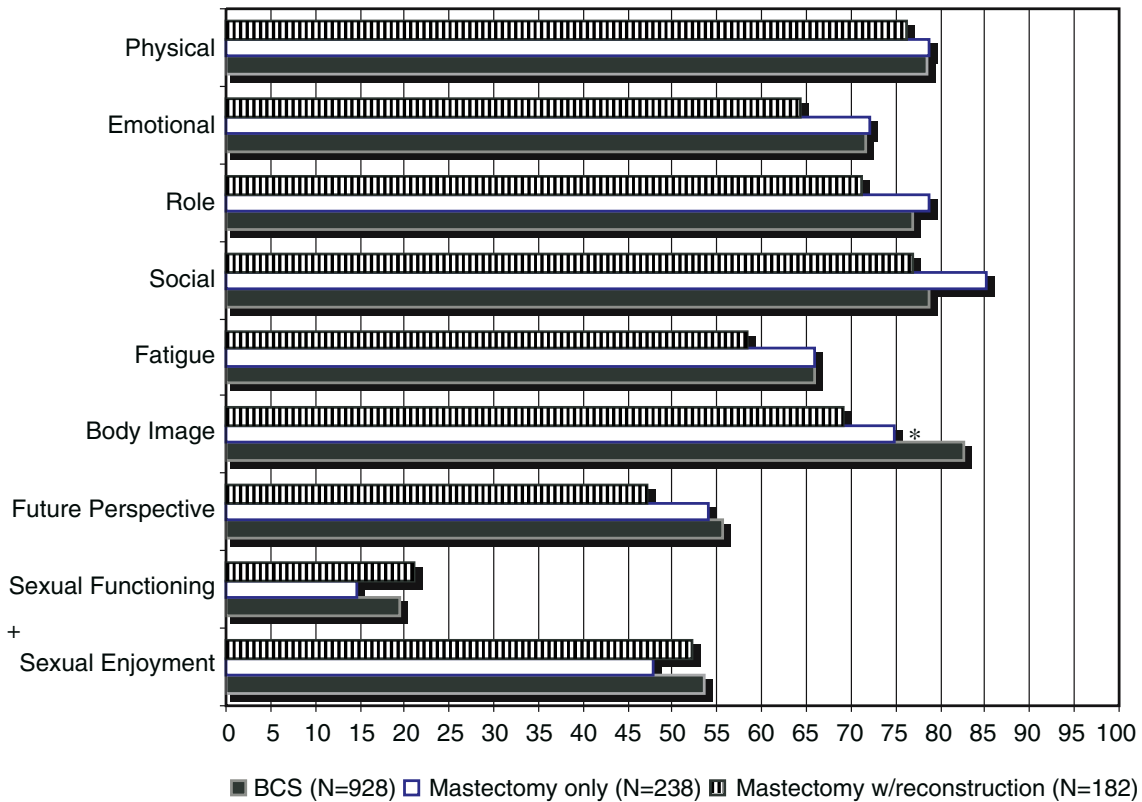

Figure 1. Mean quality of life dimension scores by primary surgical treatment. The figure controls for study site, health status at diagnosis, number of co-morbidities, response time from initial treatment, stage, chemotherapy, radiation therapy, and hormone therapy.

+ Restricted to respondents who reported being sexually active.

Statistically significant findings: $p$ value $\leqslant 0.05$ : Role Functioning, Future Perspective, Sexual Functioning; $p$ value $\leqslant 0.01$ : Emotional Functioning, Fatigue; $p$ value $\leqslant 0.001$ : Social Functioning, Body Image.

*Indicates a statistically significant and clinically meaningful difference: Body Image: BCS > Mastectomy w/reconstruction.

separated, or never married reported lower scores than respondents who were currently married.

The main-effects modeling also provides information on whether the sociodemographic factors confound the relationship among primary surgical treatment, chemotherapy, and QOL. After controlling for such factors, clinically meaningful differences dependent on surgical treatment and chemotherapy reported in Figures 1 and 2 remain for body image. However, observed differences in social functioning, fatigue, and future perspective with chemotherapy were reduced with sociodemographic factors in the model. Women who received a mastectomy with reconstruction continued to have the lowest QOL dimension scores for all outcomes (excluding sexual functioning and sexual enjoyment).

The extent to which sociodemographic factors modified the relationships among primary surgical treatment and chemotherapy with QOL was also 


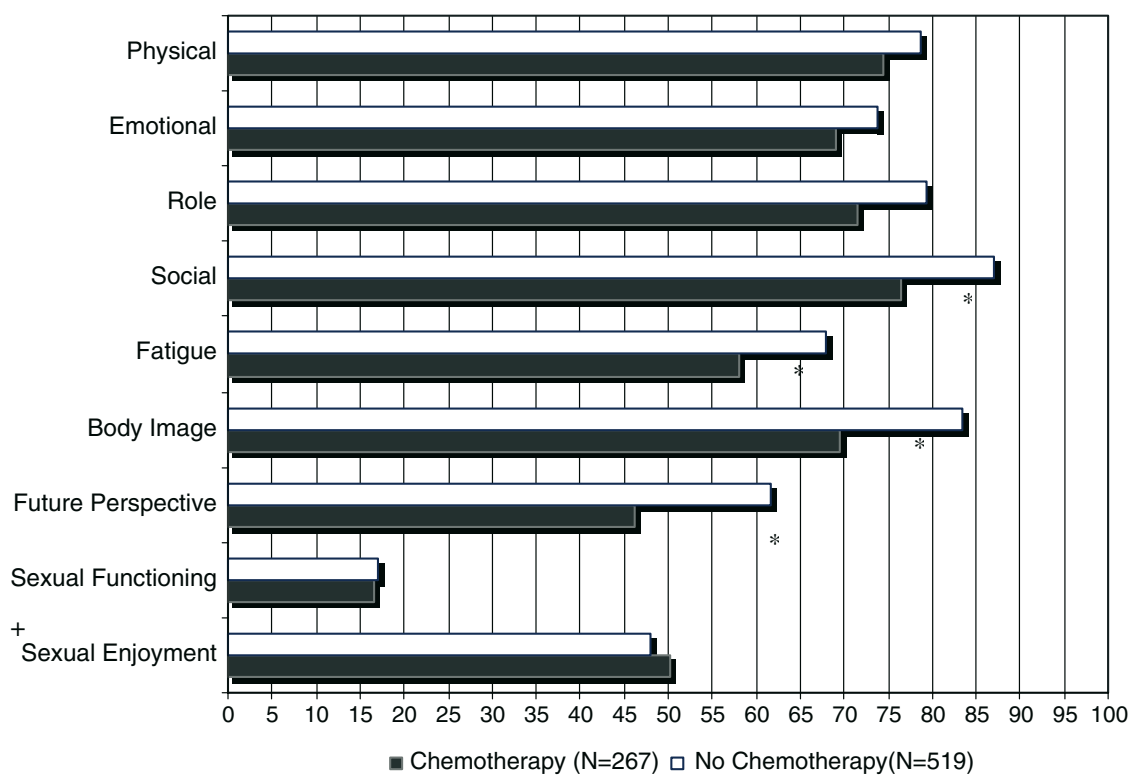

Figure 2. Mean quality of life dimension scores by receipt of chemotherapy for women with stage I or II breast disease. The figure controls for study site, health status at diagnosis, number of co-morbidities, response time from initial treatment, stage, primary surgical treatment, radiation therapy, and hormone therapy.

+ Restricted to women who reported being sexually active.

Statistically significant findings: $p$-value $<0.05$ :Physical and Emotional Functioning;

$p$ value $<0.01$ : Role Functioning; $p$ value $<0.001$ : Social Functioning, Fatigue, Body Image, Future Perspective.

*Indicates a statistically significant and clinically meaningful difference.

Note: Differences for social functioning, fatigue, and future perspective remained statistically significant but differences were less than 10 points when sociodemographic factors were added to the model.

examined. Figure 3 summarizes the significant interactions found with chemotherapy (for women with Stage I and II) and level of education. Level of education modified the relationship between chemotherapy and QOL for the functional scales excluding physical functioning. In particular, for role and social functioning, there was a gradient of higher levels of QOL with increasing levels of education for women who received chemotherapy. While emotional functioning did not have the same gradient, a trend of better QOL scores for women who received chemotherapy and had the highest level of education was observed. Additional interactions were observed for primary surgical treatment and chemotherapy for emotional functioning, social functioning, and body image (not shown). For social functioning, women who had mastectomy (with or without reconstruction) as well as chemotherapy reported higher QOL scores than women who had the combination of BCS and chemotherapy. The opposite pattern was observed for body image with BCS and chemotherapy a better combination than mastectomy and chemotherapy. No significant interactions were observed for radiation therapy.

\section{Discussion}

The results of this population-based study provide further insight into the impact of early stage breast cancer and its treatment on QOL, and the extent to which the impact differs across sociodemographic subgroups. There were no meaningful differences between cancer stage at diagnosis and QOL, independent of cancer treatment. These findings support earlier studies [3, 4] suggesting that despite the excellent prognosis for women with Stage 0, the diagnosis of breast cancer is stressful, and may result in a pattern of psychological morbidity for these women similar to that experienced by women with invasive disease.

The large sample allowed for comparisons across surgical treatment groups including BCS, mastectomy only, and mastectomy with reconstruction. 


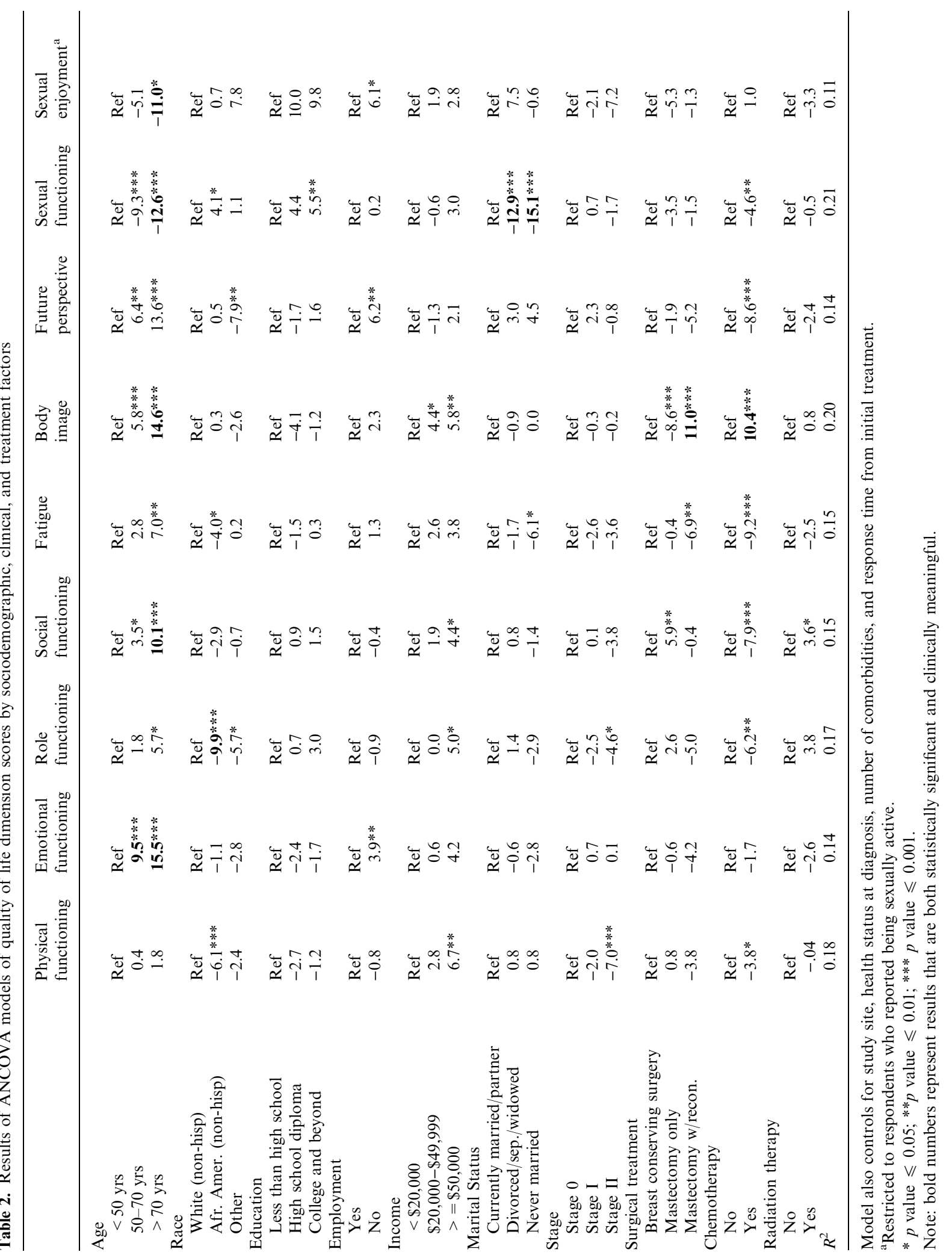




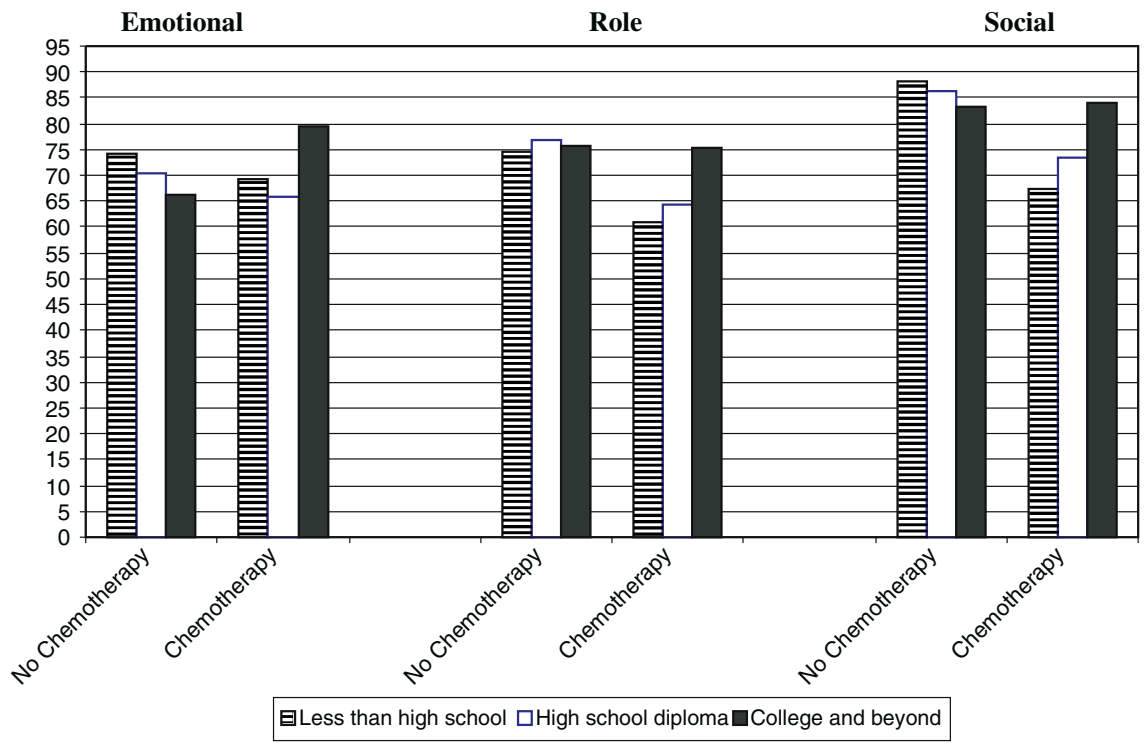

Figure 3. Mean quality of life score by receipt of chemotherapy and level of education for women with stage I, or II disease.

Means are adjusted for all other main effects including study site, age, race, employment status, income, health status at diagnosis, number of co-morbidities, response time from initial treatment, primary surgical treatment, radiation therapy, and hormone therapy Emotional functioning $p$ value $=0.001$; Role functioning $p$ value $=0.04$; Social functioning $p$ value $=0.007$.

Consistent with many previous reports $[8-10,15]$ few differences were observed across surgical options for a multitude of QOL dimensions. It appears that mastectomy only does not substantially reduce QOL compared to BCS, with the exception of body image. Women should be reassured that mastectomy is a reasonable choice in terms of both survival and major dimensions of QOL.

Similar to findings of Rowland et al. [15] when asked about body image and feeling physically less attractive, this study found QOL scores for women undergoing breast reconstruction were more similar to those receiving mastectomy only than those undergoing BCS. Although body image was the only outcome that constituted a clinically meaningful difference, women with breast reconstruction had lower QOL scores for seven QOL dimensions assessed. Other research has produced mixed results on the benefits of breast reconstruction [7, 15, 18, 38-40]. Consistent with findings of Zweiler et al. [39], women who received reconstruction reported QOL scores as high as women receiving BCS for sexual functioning and enjoyment. Another study found that women with reconstruction were the most likely to feel that breast cancer had a negative impact on their sex lives [15].
There are several possible explanations for the generally lower QOL scores observed in this study among women with breast reconstruction. QOL assessment occurred early in the treatment recovery period, and breast reconstruction may not be complete for a year or longer depending on the number of procedures required. In studies with follow-up over two years after diagnosis, Rowland et al. [15] reported differences in body image and sexuality persist, while Schover et al. [7], found no significant differences in psychosocial adjustment, body image or sexual life between women who underwent BCS compared to those with mastectomy and reconstruction. In addition, women receiving reconstruction include a subset of women who preferred BCS, but were not good candidates for the procedure. These women may have more difficulty coping with the body image and cosmetic results than women who chose mastectomy with breast reconstruction. However, women who chose reconstruction were no more likely to report decisional regret, and were slightly more satisfied with the surgical decision than women undergoing mastectomy only [41]. Nonetheless, the results suggest that women electing reconstruction should be informed of the short-term QOL impact of reconstruction that includes substantial variation 
in the length of the recovery process and uneven cosmetic results $[18,40]$.

The findings also suggest that adjuvant chemotherapy had a greater impact on QOL than which surgical procedure was performed. While the largest QOL differences were found in areas of body image and future perspective, meaningful differences were also observed in social well-being and fatigue. Schover et al. [7] also found few differences in overall psychosocial adjustment dependent on surgical choice but more psychological distress and poorer body image in women who had chemotherapy. Lower scores may be attributed to issues surrounding hair loss and malaise that persist for months after chemotherapy is completed. The onset of menopausal symptoms with chemotherapy has also been found to cause disruption in the sexual lives of younger women [42]. Contrary to previous studies supporting the negative effect of chemotherapy on sexual functioning $[9,38,43]$ this study did not find meaningful differences in sexual functioning or sexual enjoyment among women by receipt of chemotherapy.

The evidence is compelling that the impact of breast cancer on QOL is more profound in younger women. In four of nine QOL outcomes (emotional and social functioning, body image, and future perspective) younger women reported lower scores than women over 70 years of age. This disproportionate negative impact of breast cancer on younger women has been reported elsewhere [22, 23, 44-46]. Explanations for this observation include that younger women may: experience more discordance between their health expectations and their current situation resulting in feelings of greater vulnerability and impact on their QOL [21, 44]; experience greater disruptions in their daily lives, work schedules and, financial stability [44]; possess fewer coping strategies and resources to manage life-threatening illness; and, experience a menopausal transition associated with poorer health perceptions and reduced vitality [22]. Our findings cannot totally be explained by the fact that younger women may receive more aggressive treatment, since treatment was controlled for in all analyses examining sociodemographic differences.

In contrast to age-related QOL differences, few clinically important QOL differences were observed by race. Our findings are consistent with
Pikler et al. [47] but contrary to several recent studies $[20,22,23]$ suggesting that African American women may fare better than whites. African American women may utilize more religiousoriented coping and other coping strategies than whites and other ethnic groups, as well as find more meaning in life after breast cancer [22, 24, 48].

Of some concern were findings that receipt of chemotherapy had a disproportionately greater impact on women with lower levels of education. Other studies reporting a similar association between education and physical and psychosocial concerns following breast cancer [7, 49], suggest that education may provide a buffer to the negative impact of breast cancer [49]. In this study, women who reported having less than a high school diploma were also more likely to be minority (in particular, non-English speaking Hispanics), of lower income, and unemployed. Further research should explore factors that may contribute to this finding (e.g., acculturation, reading levels and cultural relevance of health communications, availability of bilingual health educators, patient-provider communication, and mistrust of the medical care system).

Study findings are limited by the crosssectional design and lack of baseline QOL assessment prior to the breast cancer diagnosis. However, health status prior to cancer diagnosis and number of co-morbidities were controlled for in all analyses. In addition, the generalizability of findings to non-white ethnic groups is somewhat compromised by the practical limitation posed by exclusion of Asian women at one site and the relatively small sample of ethnic groups outside of African Americans. Future research should actively recruit women from a variety of ethnic backgrounds to explore the extent to which ethnicity predicts QOL and/or moderates the relationships between treatment and QOL. While a degree of arbitrariness is inherent in the decision to choose a ten point difference between groups to designate a clinically meaningful difference in QOL, this decision was based on previous work by Osoba et al. [37], and avoids the pitfalls of a large sample size yielding statistically significant findings that are likely not to be clinically meaningful.

In summary, the findings from this populationbased study have implications for clinical practice 
and future research. Mastectomy and BCS are essentially equivalent choices in terms of QOL with the exception of the impact on body image. For some women, body image is a major consideration in choice of therapy, while for others concerns about recurrence or treatment duration predominate. More research is needed to sort out when the QOL benefits of breast reconstruction emerge over time, but clinicians should present both the short and long term QOL impact of breast reconstruction to patients. More research is needed to evaluate the impact of chemotherapy on QOL, especially in potentially vulnerable populations. Finally, we need to identify and target women disproportionately affected by breast cancer such as younger women and those with lower education levels, and develop behavioral and counseling interventions that address their unique issues and concerns.

\section{Acknowledgements}

This work was funded by a grant from the National Cancer Institute (R01 CA8837-A1) to the University of Michigan. This project has been funded in part with Federal funds from the Nation Cancer Institute, National Institutes of Health, Department of Health and Human Services, under Contract No. N01-PC-35139 and N01-PC-65064. The collection of cancer incidence data used in this publication was supported by the California Department of Health Services as part of the statewide cancer reporting program mandated by California Health and Safety Code Section 103885. The ideas and opinions expressed herein are those of the authors, and no endorsement by the State of California, Department of Health Services is intended or should be inferred.

\section{References}

1. Fisher B, Anderson S, Bryant J, Margolese RG, et al. Twenty year follow-up of a randomized trial comparing total mastectomy, lumpectomy, and lumpectomy plus irradiation for the treatment of invasive breast cancer. NEJM 2002; 347: 1233-1241.

2. Morrow M, Bucci C, Rademaker A. Medical contraindications are not a major factor in the underutilization of breast conserving therapy. J Am Coll Surg 1998; 186: 269-274.
3. Amichetti M, Caffo O, Arcicasa M, et al. Quality of life in patients with ductal carcinoma in situ of the breast treated with conservative surgery and postoperative irradiation. Breast Cancer Res Treat 1999; 54: 109-115.

4. Rakovitch E, Franssen E, Kim J, et al. A comparison of risk perception and psychological morbidity in women with ductal carcinoma in situ and early invasive breast cancer. Breast Cancer Res Treat 2003; 77: 285-293.

5. Shimozuma K, Ganz PA, Petersen L, Hirji K. Quality of life in the first year after breast cancer surgery: Rehabilitation needs and patterns of recovery. Breast Cancer Res Treat 1999; 56(1): 45-57.

6. Wapnir IL, Cody RP, Greco, RS. Subtle differences in quality of life after breast cancer surgery. Ann Surg Oncol 1999; 6(4): 359-366.

7. Schover LR, Yetman RJ, Tuason LJ, et al. Partial mastectomy and breast reconstruction. A comparison of their effects on psychosocial adjustment, body image, and sexuality. Cancer 1995; 75(1): 54-64.

8. de Haes JC, Curran D, Aaronson NK, Fentiman IS. Quality of life in breast cancer patients aged over 70 years, participating in the EORTC 10850 randomized clinical trial. Eur J Cancer 2003; 39(7): 945-951.

9. Ganz PA, Kwan L, Stanton AL, et al. Quality of Life at the end of primary treatment of breast cancer: First results from the Moving Beyond Cancer randomized trial. J Natl Cancer Inst 2004; 96(5): 376-387.

10. Janni W, Rjosk D, Dimpfl TH, et al. Quality of life influenced by primary surgical treatment for stage I-III breast cancer - Long term follow-up of a matched-pair analysis. Ann Surg Oncol 2001; 8(6): 542-548.

11. Moyer A. Psychosocial outcomes of breast-conserving surgery versus mastectomy: A meta-analytic review. Health Psychol 1997; 16(3): 284-298.

12. Kiebert GM, de Haes JC, van de Velde CJ. The impact of breast-conserving treatment and mastectomy on the quality of life of early-stage breast cancer patients: A review. J Clin Oncol 1991; 9(6): 1059-1070.

13. Irwig L, Bennetts A. Quality of life after breast conservation or mastectomy: A systematic review. Aust N Z J Surg 1997; 67(11): 750-754.

14. Ganz PA, Coscarelli A, Fred C, et al. Breast cancer survivors: Psychosocial concerns and quality of life. Breast Cancer Res Treat 1996; 38(2): 183-199.

15. Rowland JH, Desmond KA, et al. Role of breast reconstructive surgery in physical and emotional outcomes among breast Cancer survivors. JNCI 2000; 92(17): 1422-1429.

16. Hartl K, Janni W, Kastner R, et al. Impact of medical and demographic factors on long-term quality of life and body image of breast cancer patients. Ann Oncol 2003; 14: 1064 1071 .

17. Fallowfield LJ, Baum M, Maguire GP. Effects of breast conservation on psychological morbidity associated with diagnosis and treatment of early breast cancer. BMJ (Clinical Research Ed) 1986; 293(6558): 1331-1334.

18. Nissen MJ, Swenson KK, Ritz LJ, et al. Quality of life after breast carcinoma surgery. Cancer 2001; 91(7): 1238-1246.

19. National Institutes of Health Consensus Development Panel. National Institutes of Health Consensus 
Development Conference Statement: Adjuvant Therapy for Breast Cancer, November 1-3, 2000. J Natl Cancer Inst 2001; 93: 979-989.

20. Goldhirsch A, Glick JH, Gelber Rd, Coates AS, Senn HJ. Meeting Highlights: International Consensus Panel on the Treatment of Primary Breast Cancer. J Clin Oncol 2001; 19: 3817-3827.

21. Wenzel LB, Fairclough DL, Brady MJ, et al. Age-related differences in the quality of life of breast carcinoma patients after treatment. Cancer 1999; 86(9): 1768-1774.

22. Ganz PA, Greendale GA, Petersen L, et al. Breast cancer in younger women: Reproductive and late health effects of treatment. J Clin Oncol 2003; 21(22): 4184-4193.

23. Watters JM, Yau JC, O'Rourke K, et al. Functional status is well-maintained in older women during adjuvant chemotherapy for breast cancer. Ann Oncol 2003; 14: 1744 1750.

24. Spencer SM, Lehman JM, Wynings C, et al. Concerns about breast cancer and relations to psychosocial well-being in a multiethnic sample of early-stage patients. Health Psychol 1999; 18(2): 159-168.

25. Culver JL, Arena PL, Antoni MH, Carver CS. Coping and distress among women under treatment for early stage breast cancer: Comparing African Americans, Hispanics and non-Hispanic Whites. Psychooncology 2002; 11(6): 495-504.

26. Anema MG, Brown BE. Increasing survey responses using the total design method. J Contin Educ Nurs 1995; 26(3): $109-114$.

27. Gatz M, Harris JR, Turk-Charles S. The meaning of health for older women. In: Stanton AL, Gallant S (eds), The Psychology of Women's Health: Progress and Challenges in Research and Application. Washington, DC: American Psychological Association, 1995: 491-529.

28. Aaronson NK, Ahmedzai S, Bergman B, et al. The European Organization for Research and Treatment of Cancer QLQ-C30: A quality-of-life instrument for use in international clinical trials in oncology. J Natl Cancer Inst 1993 85(5): 365-376.

29. Sprangers MA, Groenvold M, Arraras JI, et al. The European organization for research and treatment of cancer breast cancer-specific quality-of-life questionnaire module: First results from a three-country field study. J Clin Oncol 1996; 14(10): 2756-2768.

30. Niezgoda HE, Pater JL. A validation study of the domains of the core EORTC Quality of Life Questionnaire. Qual Life Res 1993; 2: 319-325.

31. McLachlan SA, Devins GM, Goodwin PJ. Factor analysis of the psychosocial items of the EORTC QLQ-C30 in metastatic breast cancer patients participating in a psychosocial intervention study. Qual Life Res 1999; 8: 311-317.

32. Hjermstad MJ, Fossa SD, Bjordal K, Kaasa S. Test/retest study of the European organization for research and treatment of cancer core quality-of-life questionnaire. J Clin Oncol 1995; 13(5): 1249-1254.

33. Osoba D, Zee B, Pater J, et al. Psychometric properties and responsiveness of the EORTC quality of Life Questionnaire (QLQ-C30) in patients with breast, ovarian and lung cancer. Qual Life Res 1994; 3(5): 353-364.
34. Jordhoy MS, Fayers P, Loge JH, et al. Quality of life in advanced cancer patients: the impact of sociodemographic and medical characteristics. Br J Cancer 2001; 85(10): 1478-1485.

35. Kerr J, Engel J, Schlesinger-Raab A, et al. Communication, quality of life and age: Results of a 5-year prospective study in breast cancer patients. Ann Oncol 2003; 14: 421-427.

36. King, MT. The interpretation of scores from the EORTC quality of life questionnaire QLQ-C30. Qual Life Res 1996; 5: 555-567.

37. Osoba D, Rodrigues G, Myles J, Zee B, Pater J. Interpreting the significance of changes in health-related qualityof-life scores. J Clin Oncol 1998; 16(1): 139-144.

38. Arora NK, Gustafson DH, Hawkins RP, et al. Impact of surgery and chemotherapy on the quality of life of younger women with breast cancer. Cancer 2001; 92(5): 1288-1298.

39. Zweifler M, Rodriguez E, Reilly J, et al. Breast reconstruction among inner city women with breast carcinoma. Ann Plast Surg 2001; 47(1): 53-59.

40. Nissen MJ, Swenson KK, Kind EA. Quality of life after postmastectomy breast reconstruction. Oncol Nurs Forum 2002; 29(3): 547-553.

41. Lantz PM, Janz NK, Fagerlin A, et al. Satisfaction with surgery outcomes and the decision process in a populationbased sample of women with breast cancer. Health Serv Res 2004 (In Press)

42. Ganz PA, Rowland JH, Desmond K, et al. Life after breast cancer: Understanding women's health-related quality of life and sexual functioning. J Clin Oncol 1998; 16(2): 501-514.

43. Lindley C, Vasa S, Sawyer WT, Winer EP. Quality of life and preferences for treatment following systemic adjuvant therapy for early-stage breast cancer. J Clin Oncol 1998; 16(4): 1380-1387.

44. Mor V, Malin M, Allen S. Age differences in the psychosocial problems encountered by breast cancer patients. $\mathbf{J}$ Natl Cancer Inst Monogr 1994; 16: 191-197.

45. Engel J, Kerr J, Schlesinger-Raab A, et al. Predictors of Quality of Life of breast cancer patients. Acta Oncol 2003; 42: 710-718.

46. Bloom JR, Stewart SL, Johnston M, Banks P. Intrusiveness of illness and quality of life in young women with breast cancer. Psychooncology 1998; 7: 89-100.

47. Pikler V, Winterowd C. Racial and body image differences in coping for women diagnosed with breast cancer. Health Psychol 2003; 22(6): 632-637.

48. Giedzinska AS, Meyerowitz BE. Health-Related Quality of Life in a Multiethnic Sample of Breast Cancer Survivors. Ann Behav Med 2004; 28(1): 39-51.

49. King MT, Kenny P, Shiell A, et al. Quality of life three months and 1-year after first treatment for early stage breast cancer: Influence of treatment and patient characteristics. Qual Life Res 2000; 9: 789-800.

Address for correspondence: Nancy K. Janz, Department of Health Behavior and Health Education, School of Public Health, University of Michigan, 1420 Washington Heights, Ann Arbor, MI 48109-2029

Phone: + 1-734-647-3194; Fax: + 1-734-763-9115

E-mail: nkjanz@umich.edu 